\title{
Publisher's Note: Nonreciprocal electromagnetic scattering from a periodically space-time modulated slab and application to a quasisonic isolator [Phys. Rev. B 96, 165144 (2017)]
}

Sajjad Taravati, Nima Chamanara, and Christophe Caloz

(Received 16 October 2018; published 29 October 2018)

DOI: 10.1103/PhysRevB.98.159903

This paper was published online on 25 October 2017 with an incorrect Fig. 1. Figure 1 has been replaced as of 16 October 2018. The figure is incorrect in the printed version of the journal; therefore for the benefit of the print readership, the figure and its caption are replicated below.

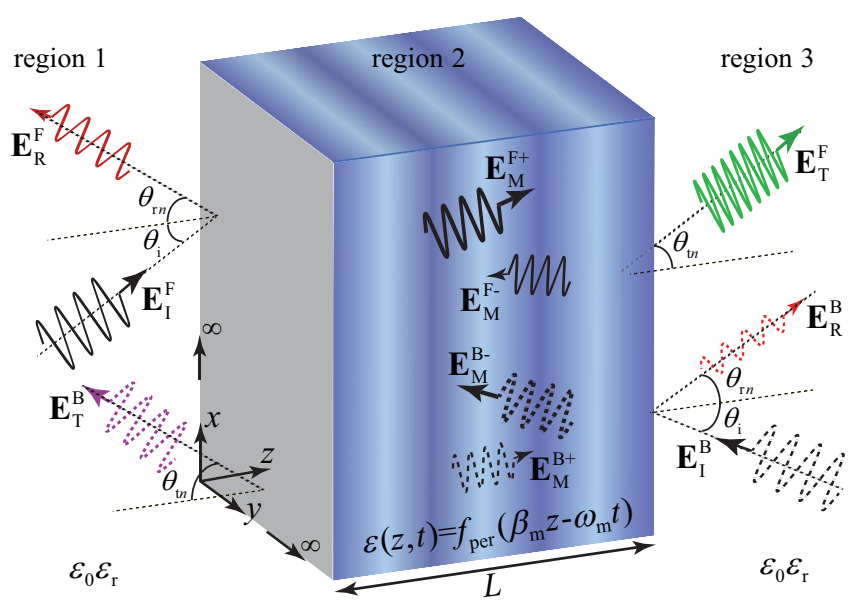

FIG. 1. Electromagnetic scattering from a periodically space-time modulated slab (region 2) sandwiched between two semi-infinite unmodulated media (regions 1 and 3). Due to the unidirectionality of the modulation, $\epsilon(z, t)=f_{\mathrm{per}}\left(\beta_{\mathrm{m}} z-\omega_{\mathrm{m}} t\right)$, the system is nonreciprocal, with different temporal and spatial frequencies scattered in the two directions. 